\title{
Cavity solitons in bidirectional lasers
}

\author{
Isabel Pérez-Arjona, Víctor J. Sánchez-Morcillo, \\ Departament de Física Aplicada, Escola Politècnica Superior de Gandia, \\ Universitat Politècnica de València, Ctra. Nazaret-Oliva S/N, 46730-Grau de Gandia, Spain \\ and Eugenio Roldán \\ Departament d'òptica, Universitat de València, Dr. Moliner 50, 46100-Burjassot, Spain
}

\begin{abstract}
We show theoretically that a broad area bidirectional laser with slightly different cavity losses for the two counterpropagating fields sustains cavity solitons (CSs). These structures are complementary, i.e., there is a bright (dark) CS in the field with more (less) losses. Interestingly, the CSs can be written/erased by injecting suitable pulses in any of the two counterpropagating fields.
\end{abstract}

Cavity solitons are localized structures that form in broad area nonlinear optical cavities in the plane orthogonal to the field propagation direction. They are interesting not only from a fundamental point of view but also because their potentialities in optical information storage and processing [1].

Interestingly, the most important nonlinear optical cavity, namely the laser, does not exhibit CSs easily, i.e., something must be done for usual lasers display localized structures different from light vortices. One possibility is introducing a suitable saturable absorber in the laser cavity [2]. Another possibility consists in rocking the laser [3], i.e., in injecting an amplitude modulated signal. These two ways have been experimentally demonstrated in lasers [4] or laser-like systems [5]. Apart from these, it has been numerically shown that cascade lasers 6], and lasers with a dense active medium [7], can sustain CSs (in the second case some intracavity spatial filtering must be done), but up to now there is no experimental evidence for these predictions.

In this letter we numerically show that CSs can form in bidirectional lasers provided that the two counterpropagating fields experience slightly different losses (up to a few per cent). Interestingly, CSs in the two fields are complementary (a bright CS in one field complements with a dark CS in the other field) and can be written/erased by acting on only one of the two fields.

Consider a broad-area ring-cavity filled with a homogeneously broadened two-level active medium which is incoherently pumped. The Maxwell-Bloch equations of this system, within the uniform field limit and rotating wave-approximations, are [8]

$$
\begin{aligned}
\partial_{t} E_{n}= & -\kappa_{n} E_{n}-i A\left\langle P_{n}\right\rangle+i a \bar{\nabla}_{\perp}^{2} E_{n} \\
\partial_{t} P_{n}= & -\gamma_{\perp}(1-i \Delta) P_{n} \\
& +i \gamma_{\perp} D\left\{E_{n}+E_{m} \exp \left[(-1)^{n} 2 i k z\right]\right\} \\
\partial_{t} D= & -\gamma_{\|}\left[D-1-\operatorname{Im}\left(E_{1} P_{1}^{*}+E_{2} P_{2}^{*}\right)\right]
\end{aligned}
$$

with $n, m=1,2$ and $n \neq m$. In the above equations $E_{n}(x, y, t)$, denote the field amplitudes of the two $(n=1,2)$ counterpropagating fields traveling along the $z$ direction, $P_{n}(x, y, z, t)$ are their respective medium polarization sources, $D(x, y, z, t)$ denotes the population in- version, and

$$
\left\langle P_{n}(x, y, t)\right\rangle=\frac{1}{L_{z}} \int_{0}^{L_{z}} d z P_{n}(x, y, z, t),
$$

are the axially-averaged medium polarizations with $L_{z}$ the laser cavity length. $\bar{\nabla}_{\perp}^{2}=\left(\partial_{x}^{2}+\partial_{y}^{2}\right)$ is the transverse Laplacian and regarding the parameters, $\Delta=$ $\left(\omega_{C}-\omega_{a t}\right) / \gamma_{\perp}$ is the normalized cavity detuning $\left(\omega_{C}\right.$ and $\omega_{a t}$ are, respectively, the cavity and atomic transition frequencies), $A$ is the pump parameter, $a$ is the diffraction coefficient, and the field, medium polarization and inversion decay rates are denoted by $\kappa_{n}, \gamma_{\perp}$, and $\gamma_{\|}$, respectively.

In order to keep the mathematical modelling as simple as possible, we shall restrict our study here to class-A lasers (i.e., those verifying $\gamma_{\perp} \gg \gamma_{\|} \gg \kappa_{1}, \kappa_{2}$ ) and shall consider perfect resonance, i.e., $\Delta=0$. For class-A lasers all material variables can be adiabatically eliminated. After taking $\partial_{t} P_{n}=\partial_{t} D=0$, one easily gets

$$
P_{n}=\frac{i\left\{E_{n}+E_{m} \exp \left[(-1)^{n} 2 i k z\right]\right\}}{1+\left|E_{1}\right|^{2}+\left|E_{2}\right|^{2}+E_{1} E_{2}^{*} e^{2 i k z}+\text { c.c. }} .
$$

Now we assume that the pump parameter value is close to its value at lasing threshold, so that field amplitudes are small quantities. Then, after Taylor expanding Eq. (3), it follows that $\left\langle P_{n}\right\rangle=i E_{n}\left(1-\left|E_{n}\right|^{2}-2\left|E_{m}\right|^{2}\right)$, and then Eqs. (1) reduce to

$$
\begin{aligned}
& \partial_{\tau} F_{1}=\left(A-1-\left|F_{1}\right|^{2}-2\left|F_{2}\right|^{2}\right) F_{1}+i \nabla_{\perp}^{2} F_{1},(4 \mathrm{a}) \\
& \partial_{\tau} F_{2}=\left(A-\sigma-\left|F_{2}\right|^{2}-2\left|F_{1}\right|^{2}\right) F_{2}+i \nabla_{\perp}^{2} F_{2},(4 \mathrm{~b})
\end{aligned}
$$

where we have introduced $F_{n}=\sqrt{A} E_{n}, \nabla_{\perp}^{2}=a \bar{\nabla}_{\perp}^{2}$, $\tau=\kappa_{1} t$, and $\sigma=\kappa_{2} / \kappa_{1}$ being $\xi=x / \sqrt{a}$ the dimensionless transverse coordinate. This is the model for a resonant class-A bidirectional laser we shall study here, which consists of a pair of coupled Ginzburg-Landau equations. It is worth mentioning that the same equations are obtained by performing a rigorous derivation based on the multiscale expansion technique, which we shall publish elsewhere. Notice that Eqs. (44) have only 
two independent parameters: pump $A$ and quotient of cavity losses $\sigma$, which we take $\sigma \geq 1$ without loss of generality.

Eqs. (44) have four possible stationary homogeneous solutions: The laser-off solution, $F_{1}=F_{2}=0$, the strong unidirectional (or singlemode) solution $\left\{F_{1}=\sqrt{A-1}, F_{2}=0\right\}$, the weak unidirectional solution $\left\{F_{1}=0, F_{2}=\sqrt{A-\sigma}\right\}$, and the bidirectional (or bimode) solution $\left\{F_{1}=(A-2 \sigma+1) / 3, F_{2}=(A-2+\sigma) / 3\right\} . \quad$ The linear stability analyses of these solutions shows that: (i), The laser-off solution losses its stability at $A=1$ giving rise to the strong unidirectional solution, which is always linearly stable; (ii), the weak unidirectional solution is linearly stable for $A \geq 2 \sigma-1$; and (iii), the bidirectional solution is always unstable. Thus bidirectional homogeneous cw emission is impossible, as it is well known from the plane-wave theory [8]. Our basic hypothesis is that stable bidirectional cw emission could be possible in a nonhomogeneous solution and this is the possibility we examine now through the numerical integration of Eqs. (4) with the purpose of finding CS solutions.

We shall concentrate here in the one-transverse dimension limit, i.e., we assume that diffraction along one transverse direction is avoided by some means, while it acts freely on the other (orthogonal) coordinate (think, e.g., of a waveguide configuration in one transverse direction). We have used a split-step algorithm for solving Eqs. (4) with a grid of 512 spatial points and a total integration length $L / \sqrt{a}=1$ with $a=10^{-5}$. In order to approach realistic conditions, here we consider that pump is limited in the transverse direction and further assume a top-hat like profile for $A$ (for computational convenience we take $A(\xi)=A \exp \left[-\left(\xi / \xi_{p}\right)^{2 n}\right]$ with $\xi_{p}=0.45$ and $n=10)$. Nevertheless, we have also carried out integrations with an unlimited pump function in order to check the robustness of our results.

We have first investigated the dynamics of the system when a real positive pulse is injected in the weak mode (its maximum value equals the weak-mode homogeneous solution), and the strong-mode homogeneous solution, with a dip arriving to zero in its center, is injected in the strong mode. Remarkably, the results are independent of the exact shape and width of the pulse (which are the same as that of the dip).

For $\sigma=1$ this initial condition relaxes to a final state in which the weak mode is null in all the space irrespective of the pump value [9]. A quite different result is reached as $\sigma$ is made different from unity and $A \geq 1.05$ : a narrow domain of emission in the weak mode, and a corresponding dip in the emission of the strong mode, are formed where the initial condition was injected, giving rise to a localized structure.

In Fig. 1 we show, in the $\langle\sigma, A\rangle$ plane, the domain of existence of these localized structures. In the inset an example of the intensity profiles of the two fields is shown. The bright CS of the weak mode is nearly Gaussian, while the dark CS -or, more precisely, the grey CS, as the minimum intensity is clearly different from zeroof the strong mode corresponds to a nearly supergaussian dip on the strong-mode homogeneous solution. The CSs represented in the inset of Fig. 1 correspond to the domain in which they are stationary but the existence of a Hopf bifurcation as $\sigma$ is decreased must be noticed. The Hopf bifurcation is supercritical, i.e. when reached (decreasing $\sigma$ ) small oscillations appear that affect both the height and width of the CS, oscillations that grow in amplitude as $\sigma$ is further decreased. In Fig. 2 an example of the dynamic behavior exhibited by the CS is shown. Fig. 2(a) shows the spatiotemporal oscillation of the two fields, the maximum of weak field, $F_{2}$, exhibits periodic oscillations, see Fig. 2(b).

The continuous line in Fig. 1 corresponds to the boundary where the CSs cease to exist. When crossing this line, the bright CS of the weak mode simply decreases in amplitude, quickly, till it disappears, giving rise to homogeneous emission in the strong mode. Something similar occurs at $\sigma=1$, where the oscillating CSs collapses.

After establishing the existence of these CSs, an important issue is to determine whether they can be manipulated, i.e., how can they be written/erased. Notice that in the bidirectional laser one can inject signals in either the strong or the weak mode or in both. The results we have just commented are obtained from an initial condition which, to some extent, can be understood as the simultaneous injection of a positive pulse in the weak mode and a negative pulse in the strong one. But from an experimental perspective this could be difficult to implement. Moreover, one must take into account the unavoidable presence of noise in real devices.

We have thus carried out a series of numerical integrations in which we have tested the writing and erasing of CSs by injecting appropriate (i.e., with the right phase) pulses in only one of the two counterpropagating fields in the presence of additive noise (random noise with amplitude $10^{-5}$ ) in Eqs. (4). The results are summarized in Fig. 3. The initial condition consists of emission only in the strong mode. We start with homogeneous steady state emission in the strong mode. At $\tau=0$, a positive Gaussian pulse is added only in the weak mode for a short time, the result being the formation of the CSs. The CSs are then removed by injecting a positive Gaussian in the strong mode, at $\tau=2 \cdot 10^{4}$. Then, at $\tau=2.5 \cdot 10^{4}$ a negative Gaussian is injected in the strong mode, and the CSs are formed again, which are later removed by injecting again at $\tau=3.5 \cdot 10^{4}$ a Gaussian pulse in the weak field but with the opposite phase (the injection times are marked in the figure with a horizontal white line). These numerical results prove the robustness and addresability of these localized structures.

We have also assessed the possibility that the system supports clusters formed by an array of CSs. The numerical results ensured that several CSs can be switched on 
and that this solution is stable and robust in the presence of noise. Figure 4 shows the spatiotemporal evolution where a cluster formed by four CSs is developed (a) from the strong mode emission initial condition when four Gaussian pulses are injected in the weak field and the final intensity profile (b).

In conclusion we have shown that cavity solitons form in class-A bidirectional lasers when the two counterpropagating fields experience slightly different cavity losses. We find that it is particularly remarkable the fact that (i) the CSs in the two fields are complementary, i.e., when one field exhibits a bright CS, the counterpropagating field shows a dark CS in the same position, and (ii) the CSs can be written/erased by acting on only one of the two fields.

This work has been supported by the Spanish Ministerio de Educación y Ciencia and the European Union FEDER through Projects FIS2005-07931-C03-01 and -02 and Programa Juan de la Cierva.
${ }^{1}$ K. Staliunas and V.J. Sánchez-Morcillo, Transverse Patterns in Nonlinear Optical Resonators, (Springer, Berlín, 2003).

2 N. Rosanov, Prog. Opt. 35, 1 (1996).

3 G.J. de Valcárcel and K. Staliunas, Phys. Rev. E 67, 026604 (2003).

${ }^{4}$ V.B. Taranenko, K. Staliunas, and C.O. Weiss, Phys. Rev. A 56, 1582 (1997).

5 A. Esteban-Martín, M. Martínez-Quesada, V.B. Taranenko, E. Roldán, and G.J. de Valcárcel, Phys. Rev. Lett. 97, 093903 (2006).

${ }^{6}$ R. Vilaseca, M. C. Torrent, J. García-Ojalvo, M. Brambilla, and M. San Miguel, Phys. Rev. Lett. 87, 083902 (2001).

7 V. Ahufinger, J. García-Ojalvo, J. Mompart, M. C. Torrent, R. Corbalán, and R. Vilaseca, Phys. Rev. Lett. 91, 083901 (2003).

8 H. Zeghlache, P. Mandel, N. B. Abraham, L. M. Hoffer, G. L. Lippi, and T. Mello, Phys. Rev. A 37, 470 (1988).

${ }^{9}$ When the pump transverse extension is unlimited, the dynamics is different: one observes that two complementary domains of emission are formed in the two fields (where one mode is on the other mode is off and viceversa) that oscillate expanding and contracting periodically. This behavior disappears when the pump transverse extension is finite.

\section{FIGURE CAPTIONS}

Fig.1. Bifurcation diagram of CSs in the $\langle\sigma, A\rangle$ plane. Continuous and dashed lines represent the boundaries of existence of stationary and dynamic CSs, respectively. The inset shows a stationary CS obtained for $\sigma=1.01$ and $A=1.4$. Continuous (dashed) line corresponds to the field amplitudes in the weak (strong) modes.

Fig. 2. Pulsing CS obtained for $\sigma=1.005$ and $A=$ 1.4. The spatiotemporal evolution of the pulsing CS is shown in (a), where $\tau$ runs from $2.910^{5}$ to $310^{5}$ and $\xi$ from -0.5 to 0.5 , the maximum of the CS in the weak field oscillates periodically in time (b).

Fig. 3. Switching on and off of CSs by different techniques. Parameters are $\sigma=1.015$ and $A=1.4, \xi$ running from -0.5 to 0.5 . The white lines indicate the times at which pulses are injected. For details, see text.

Fig. 4. Cluster formed by four CSs for $\sigma=1.005$ and $A=1.4$. Time $\tau$ runs from 0 to $2.510^{4}$. Spatiotemporal evolution (a) from the injection and final intensity profile (b). 


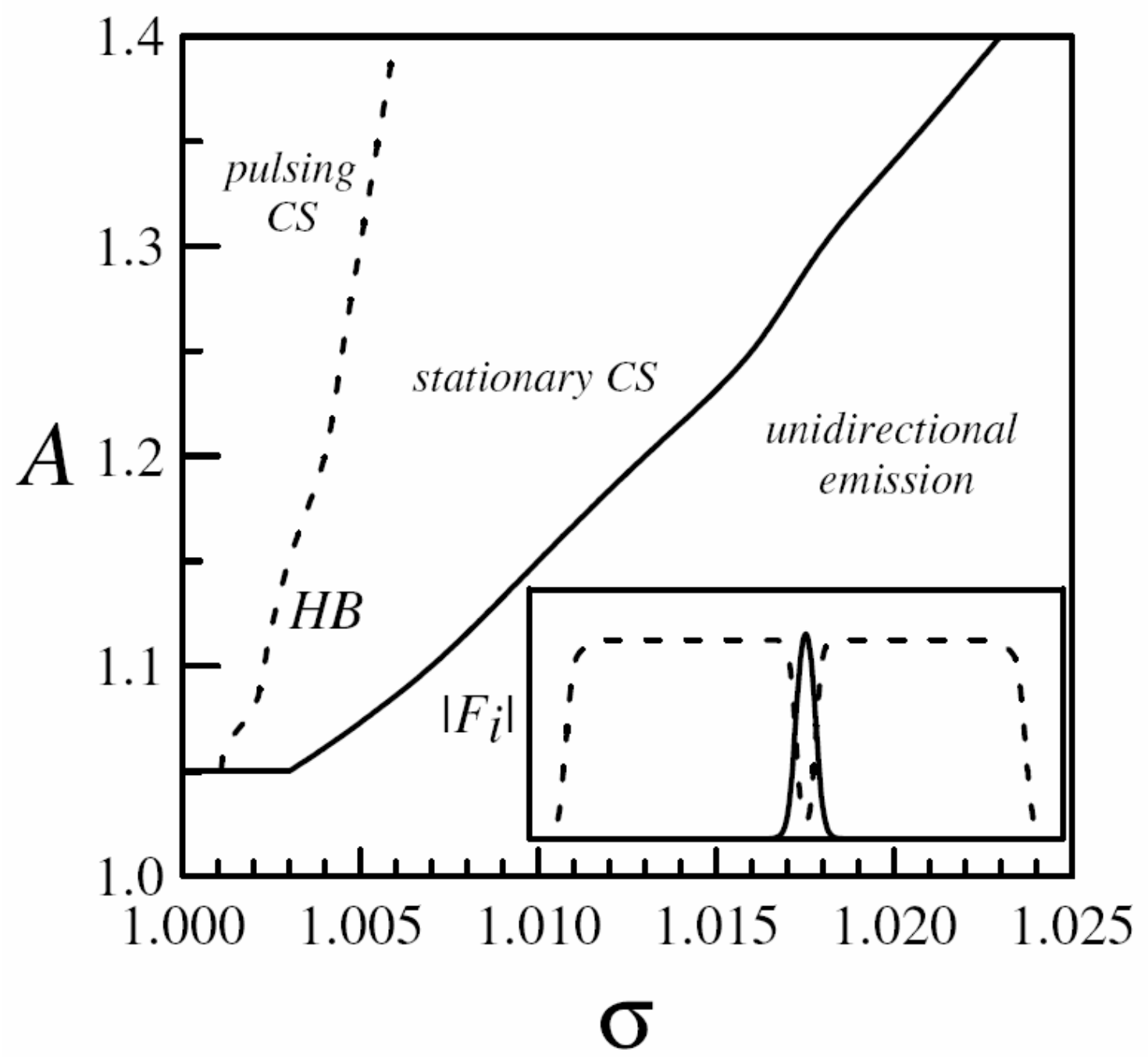

Fig. 1 Pérez-Arjona et al. 


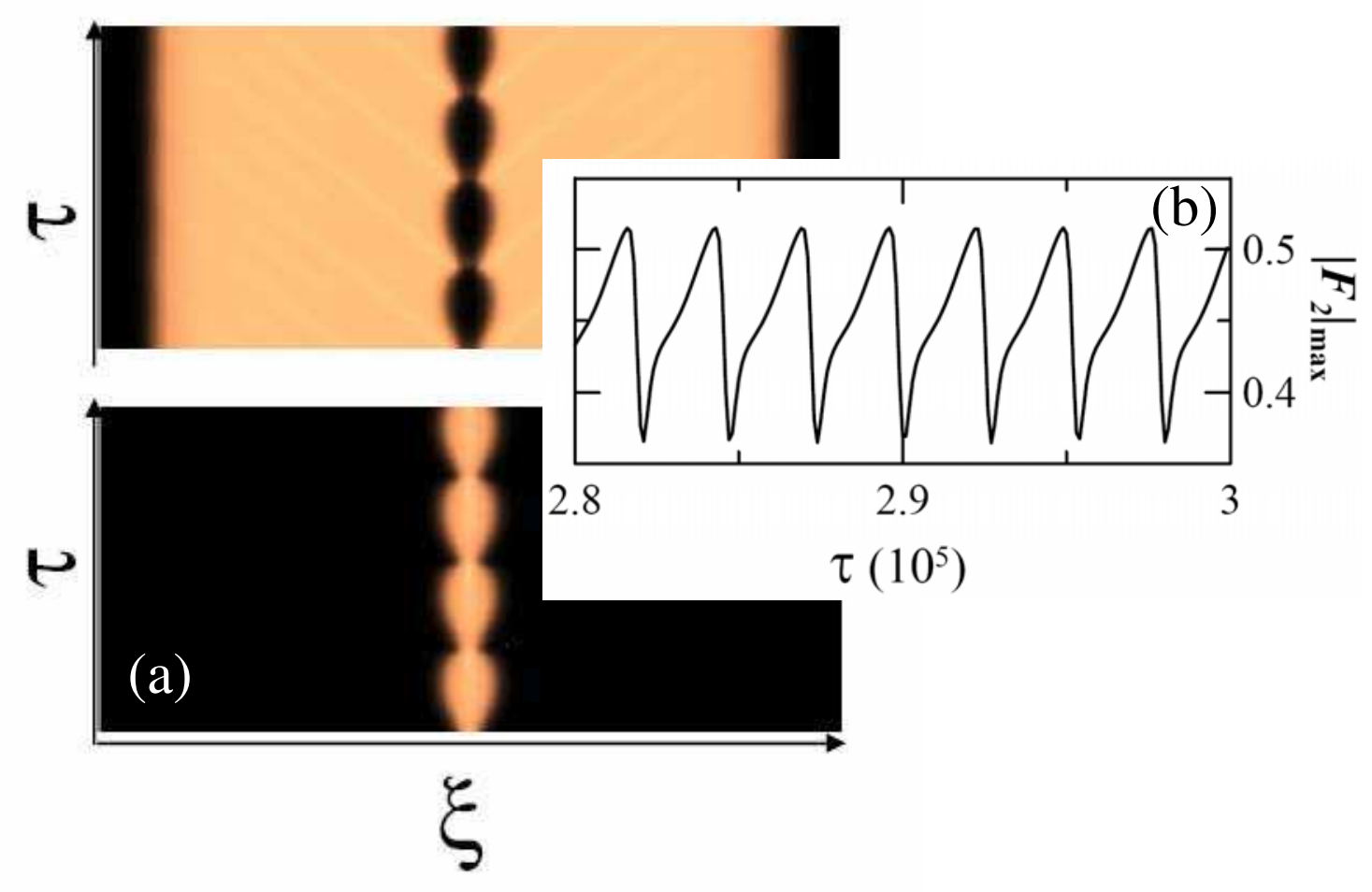

Fig. 2 Pérez-Arjona et al. 

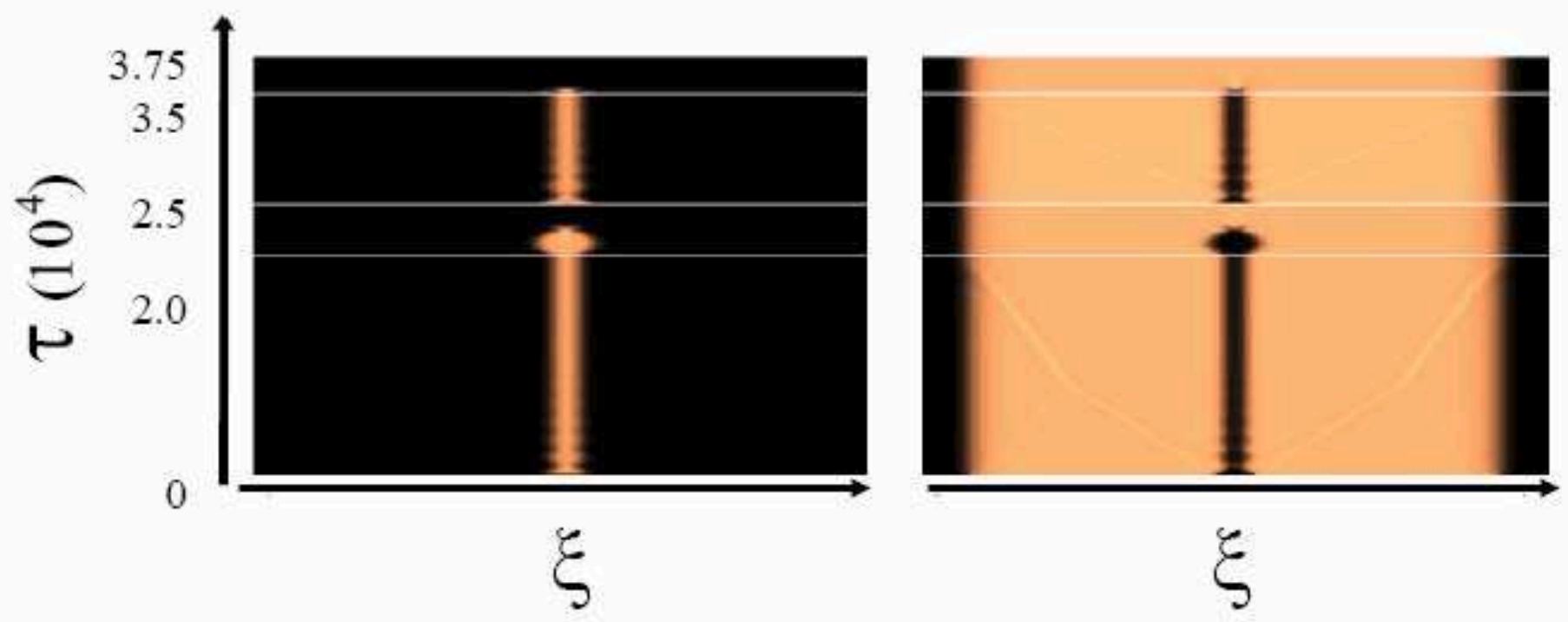

Fig. 3 Pérez-Arjona et al. 


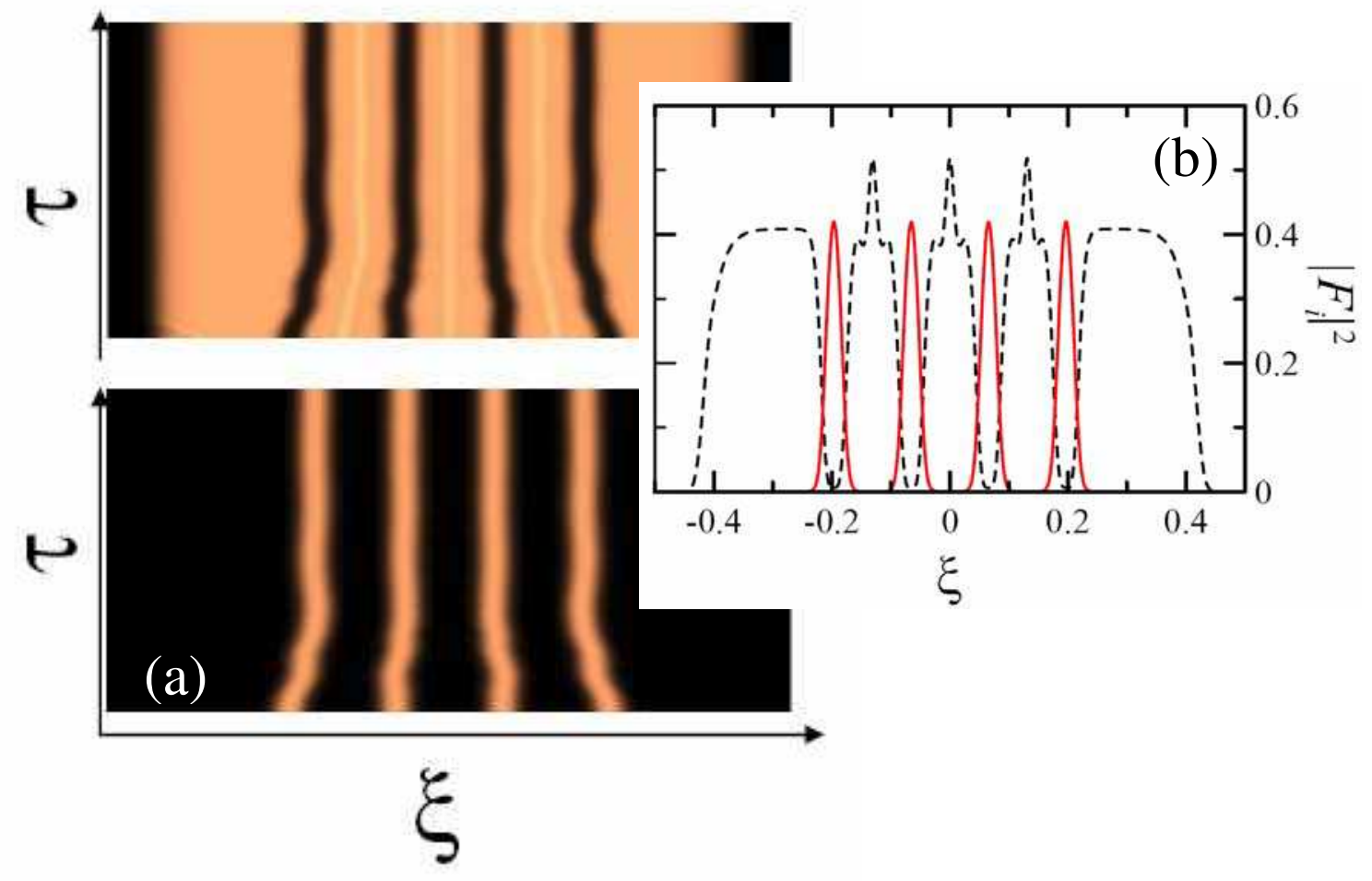

Fig. 4 Pérez-Arjona et al. 